\title{
Two-Way and Multiple-Access Energy Harvesting Systems with Energy Cooperation
}

\author{
Berk Gurakan ${ }^{1}$, Omur Ozel ${ }^{1}$, Jing Yang ${ }^{2}$, and Sennur Ulukus ${ }^{1}$ \\ ${ }^{1}$ Department of Electrical and Computer Engineering, University of Maryland, College Park, MD 20742 \\ ${ }^{2}$ Department of Electrical Engineering, University of Arkansas, Fayetteville, AR 72701
}

\begin{abstract}
We study the capacity regions of two-way and multiple-access energy harvesting communication systems with one-way wireless energy transfer. In these systems, energy required for data transmission is harvested by the users from nature throughout the communication duration, and there is a separate unit that enables energy transfer from the first user to the second user with an efficiency of $\alpha$. Energy harvests are known by the transmitters a priori. We first investigate the capacity region of the energy harvesting Gaussian two-way channel (TWC) with one-way energy transfer. We show that the boundary of the capacity region is achieved by a generalized two-dimensional directional water-filling algorithm. Then, we study the capacity region of the energy harvesting Gaussian multiple access channel (MAC) with one-way energy transfer. We show that if the priority of the first user is higher, then energy transfer is not needed. In addition, if the priority of the second user is sufficiently high, then the first user must transfer all of its energy to the second user.
\end{abstract}

\section{INTRODUCTION}

We study the capacity regions of the Gaussian two-way channel (TWC) and the Gaussian two-user multiple access channel (MAC) with one-way energy transfer. In both scenarios, there are two users powered by energy harvesting devices communicating messages to each other or to an access point. We model these scenarios as two users having exogenous energy arrival processes that recharge their batteries throughout the communication duration. Additionally, oneway energy transfer is possible: The first user can transmit a portion of its energy to the second user through a separate wireless energy transfer unit subject to an inefficiency (i.e., loss) during the transfer. Wireless energy transfer enables a new form of cooperation which we call energy cooperation; see also [1]. In contrast to the usual notion of cooperation, which is at the signal level [2], energy cooperation is at the battery energy level.

In this paper, we study optimal energy management policies for the users in systems with energy cooperation. Assuming that the users know the realizations of the energy arrival processes in advance, as in the existing literature [1], [3][14], we characterize the corresponding capacity regions. We first consider the Gaussian TWC with energy transfer. We show that the boundary of the capacity region is obtained by a generalized two-dimensional directional water-filling

This work was supported by NSF Grants CNS 09-64632, CCF 09-64645, CCF 10-18185 and CNS 11-47811.

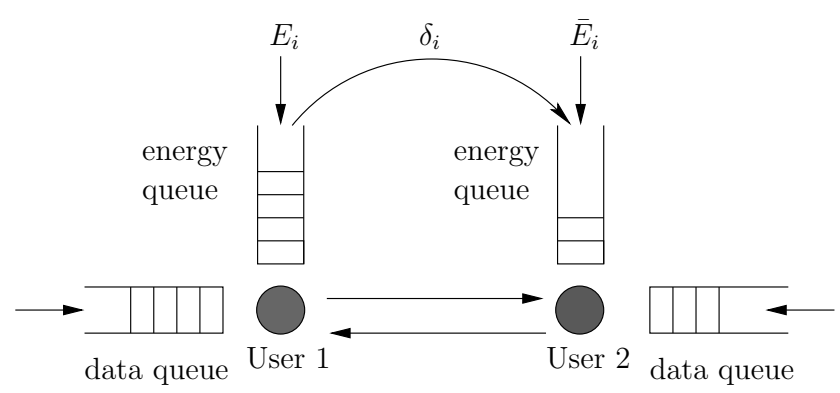

Fig. 1. TWC with one-way energy transfer.

algorithm. This algorithm optimizes the energy levels in two dimensions, namely the time and user dimensions, subject to energy causality constraints. We then study the Gaussian MAC with energy transfer. We show that if the first user has a higher priority, then energy transfer is not needed and the boundary is achieved by the generalized backward directional water-filling algorithm given in [9]. Moreover, we show that if the second user has a sufficiently high priority, then transferring all of the energy of the first user to the second user is optimal. In between these two extremes, some non-zero portion of the first user's energy is transferred to the second user.

\section{TWC WITH ONE-WAY ENERGY TRANSFER}

We consider a Gaussian TWC as shown in Fig. 1. The two queues at the nodes are the data and energy queues. The energies that arrive at the nodes are saved in the corresponding energy queues. The data queues of both users always carry some data packets. The physical layer is a memoryless Gaussian TWC [15] where the channel inputs and outputs are $x_{1}, x_{2}$ and $y_{1}, y_{2}$, respectively. The input-output relations are $y_{1}=x_{1}+x_{2}+n_{1}$ and $y_{2}=x_{1}+x_{2}+n_{2}$ where $n_{1}$ and $n_{2}$ are independent Gaussian noises with zero-mean and unitvariance. We assume that the time is slotted and there are a total of $T$ equal length slots. In slot $t$, the first and second users harvest energy in amounts $E_{t}$ and $\bar{E}_{t}$, respectively.

There is a separate one-way wireless energy transfer unit from the first user to the second user with efficiency $0 \leq$ $\alpha \leq 1$ : When the first user transfers $\delta_{i}$ amount of energy to the second user, $\delta_{i}$ amount of energy exits the first user's energy queue and $\alpha \delta_{i}$ amount of energy enters the second user's energy queue in the same slot. The power policy of user 
1 is composed of the sequences $\mathbf{P}, \boldsymbol{\delta}$, and the power policy of user 2 is the sequence $\overline{\mathbf{P}}$.

For both users, the energy that has not arrived yet cannot be used for data transmission or energy transfer. In addition, energy transfer amounts cannot be larger than the harvested energy. These constraints yield the following set $\mathcal{F}$ of feasible power control and energy transfer policies:

$$
\begin{aligned}
\mathcal{F}=\{(\boldsymbol{\delta}, \mathbf{P}, \overline{\mathbf{P}}): \quad & \sum_{i=1}^{k} P_{i} \leq \sum_{i=1}^{k}\left(E_{i}-\delta_{i}\right), \quad \forall k \\
& \sum_{i=1}^{k} \bar{P}_{i} \leq \sum_{i=1}^{k}\left(\bar{E}_{i}+\alpha \delta_{i}\right), \quad \forall k \\
& \left.\sum_{i=1}^{k} \delta_{i} \leq \sum_{i=1}^{k} E_{i}, \quad \forall k\right\}
\end{aligned}
$$

For the Gaussian TWC with individual power constraints $P_{1}$ and $P_{2}$, rate pairs $\left(R_{1}, R_{2}\right)$ with $R_{1} \leq \frac{1}{2} \log \left(1+P_{1}\right), R_{2} \leq$ $\frac{1}{2} \log \left(1+P_{2}\right)$ are achievable [15]. For a fixed energy transfer vector $\delta$, and feasible power control policies $\mathbf{P}$ and $\overline{\mathbf{P}}$, the set of achievable rates is:

$$
\begin{aligned}
\mathcal{C}_{\boldsymbol{\delta}}(\mathbf{P}, \overline{\mathbf{P}})=\left\{\left(R_{1}, R_{2}\right):\right. & R_{1} \leq \sum_{i=1}^{T} \frac{1}{2} \log \left(1+P_{i}\right) \\
R_{2} & \left.\leq \sum_{i=1}^{T} \frac{1}{2} \log \left(1+\bar{P}_{i}\right)\right\}
\end{aligned}
$$

The notation shows the dependence of the region on the energy transfer vector $\delta$. This region is shown in Fig. 2 for different values of $\delta$. Each of these regions are rectangles of the form $R_{i} \leq C_{i}$ where $C_{i}$ is the maximum throughput achieved for user $i$ found by maximizing (2) constrained to the feasibility constraints $\mathcal{F}$. As $\delta$ is increased, energy is transferred from user 1 to user 2 therefore $C_{1}$ decreases while $C_{2}$ increases. By taking the union of the regions over all possible energy transfer vectors and power policies for the users, we obtain the capacity region of the Gaussian TWC as:

$$
\mathcal{C}(\mathbf{E}, \overline{\mathbf{E}})=\bigcup_{(\delta, \mathbf{P}, \overline{\mathbf{P}}) \in \mathcal{F}} \mathcal{C}_{\delta}(\mathbf{P}, \overline{\mathbf{P}})
$$

\section{CAPACITy REgion OF the GaUsSian TWC}

In this section, we characterize the capacity region as well as the optimal power allocation and energy transfer policies. We start by noting that the capacity region is convex.

Lemma $1 \mathcal{C}(\boldsymbol{E}, \overline{\boldsymbol{E}})$ is a convex region.

Since $\mathcal{C}(\mathbf{E}, \overline{\mathbf{E}})$ is convex, each boundary point can be found by solving the following weighted rate maximization problem:

$$
\begin{aligned}
\max _{\bar{P}_{i}, P_{i}, \delta_{i}} & \sum_{i=1}^{T} \theta_{1} \frac{1}{2} \log \left(1+P_{i}\right)+\theta_{2} \frac{1}{2} \log \left(1+\bar{P}_{i}\right) \\
\text { s.t. } & (\boldsymbol{\delta}, \mathbf{P}, \overline{\mathbf{P}}) \in \mathcal{F}
\end{aligned}
$$

The problem in (4) is a convex optimization problem as the objective function is concave and the feasible set is a convex

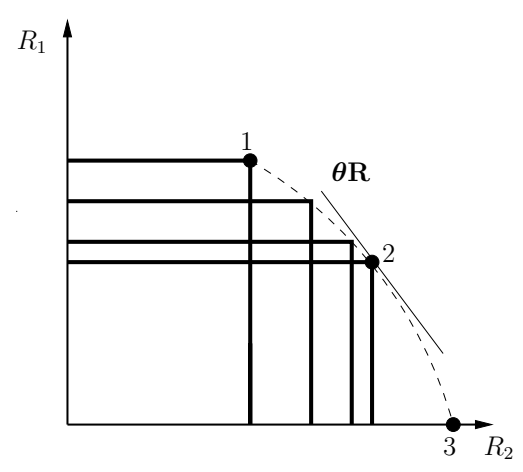

Fig. 2. Capacity region of the Gaussian TWC.

set [16]. We write the Lagrangian function for (4) as

$$
\begin{aligned}
\mathcal{L}= & -\sum_{i=1}^{T} \theta_{1} \log \left(1+P_{i}\right)+\theta_{2} \log \left(1+\bar{P}_{i}\right) \\
& +\sum_{k=1}^{T} \mu_{k}\left(\sum_{i=1}^{k} P_{i}-\left(E_{i}-\delta_{i}\right)\right) \\
& +\sum_{k=1}^{T} \eta_{k}\left(\sum_{i=1}^{k} \bar{P}_{i}-\left(\bar{E}_{i}+\alpha \delta_{i}\right)\right) \\
& +\sum_{k=1}^{T} \gamma_{k}\left(\sum_{i=1}^{k} \delta_{i}-E_{i}\right)-\sum_{k=1}^{T} \rho_{k} \delta_{k}
\end{aligned}
$$

The Lagrange multiplier $\rho_{k}$ is due to the constraint $\delta_{k} \geq 0$. We exclude the non-negativity constraints for $P_{i}$ and $\bar{P}_{i}$ as $P_{i}$ and $\bar{P}_{i}$ are always non-zero in the optimal policy for $\theta_{1}, \theta_{2}>0$. Similarly, we eliminate the constraints $\sum_{i=1}^{k} \delta_{i} \leq \sum_{i=1}^{k} E_{i}$ and the multipliers $\gamma_{k}$ in the following analysis since these constraints can never be satisfied with equality in the optimal policy for the Gaussian TWC for $\theta_{1}, \theta_{2}>0$, as that would require $P_{i}=0$ for some $i$. However, we note that these constraints and the multipliers $\gamma_{k}$ play an important role for the analysis of the capacity region of the MAC and hence we reinstate these constraints when necessary. The KKT conditions for the case of TWC are:

$$
\begin{aligned}
-\frac{\theta_{1}}{1+P_{i}}+\sum_{k=i}^{T} \mu_{k} & =0, \quad \forall i \\
-\frac{\theta_{2}}{1+\bar{P}_{i}}+\sum_{k=i}^{T} \eta_{k} & =0, \quad \forall i \\
\sum_{k=i}^{T} \mu_{k}-\alpha \sum_{k=i}^{T} \eta_{k}-\rho_{i} & =0, \quad \forall i
\end{aligned}
$$

with the additional complementary slackness conditions as:

$$
\begin{aligned}
\mu_{k}\left(\sum_{i=1}^{k} P_{i}-\left(E_{i}-\delta_{i}\right)\right) & =0, \quad \forall k \\
\eta_{k}\left(\sum_{i=1}^{k} \bar{P}_{i}-\left(\bar{E}_{i}+\alpha \delta_{i}\right)\right) & =0, \quad \forall k \\
\rho_{k} \delta_{k} & =0, \quad \forall k
\end{aligned}
$$


From (6), (7) and (8) we get:

$$
\begin{aligned}
P_{i} & =\frac{\theta_{1}}{\sum_{k=i}^{T} \mu_{k}}-1, \quad \forall i \\
\bar{P}_{i} & =\frac{\theta_{2}}{\sum_{k=i}^{T} \eta_{k}}-1, \quad \forall i \\
\rho_{i} & =\sum_{k=i}^{T} \mu_{k}-\alpha \sum_{k=i}^{T} \eta_{k}, \quad \forall i
\end{aligned}
$$

We will give the solution for general $\theta_{1}, \theta_{2}>0$ in the sequel. Before that, we note that in the extreme case when $\theta_{2}=0$, the problem reduces to maximizing the first user's throughput only and hence any energy transfer is strictly suboptimal, i.e., $\boldsymbol{\delta}=\mathbf{0}$ is optimal. This corresponds to point 1 in Fig. 2. Similarly, when $\theta_{1}=0$, the problem reduces to maximizing the second user's throughput only and the first user must transfer all of its energy to the second user, i.e., $\boldsymbol{\delta}=\mathbf{E}$ is optimal. This corresponds to point 3 in Fig. 2. When $\theta_{1}, \theta_{2}>0$, we obtain the points between points 1 and 3 in Fig. 2. In this case, for a given energy transfer profile $\delta_{1}, \ldots, \delta_{T}$, the optimization problem can be separated into two optimization problems, each only in terms of the power control policy of the corresponding user.

Lemma 2 The optimal power sequences $P_{i}^{*}$ and $\bar{P}_{i}^{*}$ are monotonically increasing sequences: $P_{i+1}^{*} \geq P_{i}^{*}, \bar{P}_{i+1}^{*} \geq \bar{P}_{i}^{*}$.

Next, we provide the necessary optimality condition for a non-zero energy transfer.

Lemma 3 For the optimal power sequences $P_{i}^{*}, \bar{P}_{i}^{*}$ and energy transfer sequence $\delta_{i}^{*}$, if $\delta_{i}^{*} \neq 0$ for a slot $i$, then,

$$
\frac{1+P_{i}^{*}}{1+\bar{P}_{i}^{*}}=\frac{\theta_{1}}{\theta_{2} \alpha}
$$

Proof: From (12)-(14) we have

$$
\frac{1+P_{i}^{*}}{1+\bar{P}_{i}^{*}}=\frac{\theta_{1} \sum_{k=i}^{T} \eta_{k}}{\theta_{2}\left(\alpha \sum_{k=i}^{T} \eta_{k}+\rho_{i}\right)}
$$

If there is a non-zero energy transfer, $\delta_{i} \neq 0$, we have from (11), $\rho_{i}=0$. Therefore, (15) must be satisfied if $\delta_{i} \neq 0$.

In order to devise an algorithmic solution, we apply a change of variable $\tilde{P}_{i}=\frac{\vec{P}_{i}}{\alpha}$ and re-write the optimization problem in terms of $P_{i}, \tilde{P}_{i}, \delta_{i}$ as follows:

$$
\begin{aligned}
\max _{\tilde{P}_{i}, P_{i}, \delta_{i}} & \sum_{i=1}^{T} \theta_{1} \frac{1}{2} \log \left(1+P_{i}\right)+\theta_{2} \frac{1}{2} \log \left(1+\alpha \tilde{P}_{i}\right) \\
\text { s.t. } \quad & \sum_{i=1}^{k} P_{i} \leq \sum_{i=1}^{k}\left(E_{i}-\delta_{i}\right), \quad \forall k \\
& \sum_{i=1}^{k} \tilde{P}_{i} \leq \sum_{i=1}^{k}\left(\frac{\bar{E}_{i}}{\alpha}+\delta_{i}\right), \quad \forall k \\
& \sum_{i=1}^{k} \delta_{i} \leq \sum_{i=1}^{k} E_{i}, \quad \forall k
\end{aligned}
$$

The optimal power allocation for this problem is:

$$
\begin{aligned}
& P_{i}^{*}=\theta_{1} \nu_{i}-1, \quad \forall i \\
& \tilde{P}_{i}^{*}=\theta_{2} \tilde{\nu}_{i}-\frac{1}{\alpha}, \quad \forall i
\end{aligned}
$$

where $\nu_{i}$ and $\tilde{\nu}_{i}$ in slot $i$ are defined by

$$
\nu_{i}=\frac{1}{\sum_{k=i}^{T} \mu_{k}} \quad \text { and } \quad \tilde{\nu}_{i}=\frac{1}{\sum_{k=i}^{T} \eta_{k}}
$$

The power level expressions in (18)-(19) lead to a directional water-filling interpretation [5]. In particular, we note that energy has to be jointly allocated in time and user dimensions together. This calls for a two-dimensional directional waterfilling algorithm where energy is allowed to flow in two dimensions, from left to right (in time) and from up to down (among users). We utilize right permeable taps to account for energy which will be used in the future and down permeable taps to account for energy that will be transferred from user 1 to user 2 . We see from the KKT optimality conditions that $\nu_{i}=\tilde{\nu}_{i}$ in slots where there is non-zero energy transfer. We note that in the original problem, this implies that if some energy is transferred, then the power levels in that slot need to satisfy (15). The base levels for users 1 and 2 are 1 and $\frac{1}{\alpha}$, respectively. Moreover, to facilitate the water flow interpretation, we scale the energy arrivals of user 2 by $\frac{1}{\alpha}$ as seen in (17). If the resulting water levels are higher for user 1 or not monotonically increasing in time for both users, then water has to flow until the levels are balanced.

While finding the balanced water levels, the two dimensions of the water flow (i.e., in time and among users) are coupled and therefore it is not easy to determine beforehand which taps will be open or closed in the optimal solution. In particular, the water flow of user 2 from time slot $i$ to time slot $i+j$, $j>0$, may become redundant if some energy is transferred from user 1. To circumvent this difficulty, we let each tap (right/down permeable) have a meter measuring the water that has already passed through it and we allow that tap to let the water flow back if an update in the allocation necessitates it. This way, we keep track of the source of the energy and whether it is transferred to future time slots or to the other user. First, we fill energy into the slots with all taps closed. Then, we open only the right permeable taps and perform directional water-filling for both users individually [5]. Then, we open the down taps one by one in a backward fashion. If water flows down through a tap, the amount is measured by the meter. Water levels in the slots connected by the bidirectional horizontal taps need to be equal. Whenever water flows down through a down permeable tap, the water levels must satisfy the proportionality relationship in (15). When the water levels are properly balanced, the optimal solution is obtained.

\section{MAC WITH ONE-WAY ENERGY TRANSFER}

In this section, we consider the MAC scenario as shown in Fig. 3. In MAC, the received signal is $y=x_{1}+x_{2}+n$ where $x_{1}$ and $x_{2}$ are signals of user 1 and user 2, respectively, and $n$ is a Gaussian noise with zero-mean and unit-variance. For the 


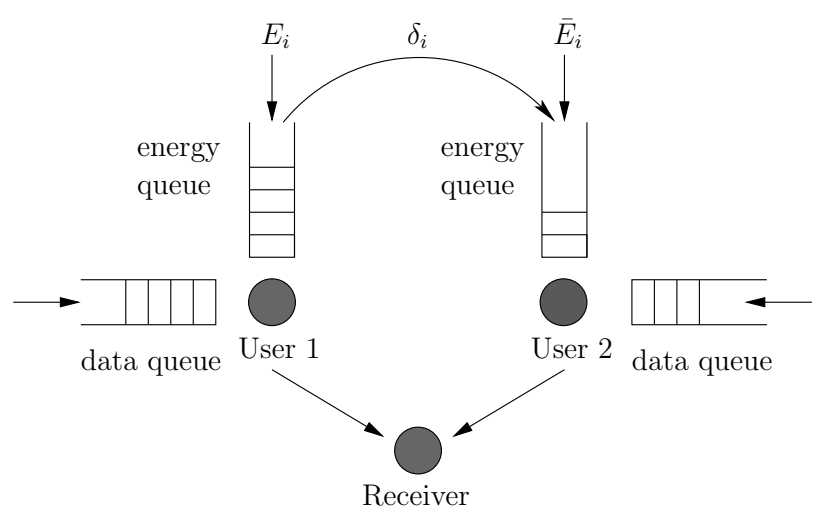

Fig. 3. MAC with one-way energy transfer.

Gaussian two-user MAC with individual power constraints $P_{1}$ and $P_{2}$, rate pairs $\left(R_{1}, R_{2}\right)$ with $R_{1} \leq \frac{1}{2} \log \left(1+P_{1}\right), R_{2} \leq$ $\frac{1}{2} \log \left(1+P_{2}\right), R_{1}+R_{2} \leq \frac{1}{2} \log \left(1+P_{1}+P_{2}\right)$ are achievable [17]. For a fixed energy transfer vector $\delta$, and feasible power control policies $\mathbf{P}$ and $\overline{\mathbf{P}}$, the set of achievable rates is a pentagon defined as [9]:

$$
\begin{aligned}
\mathcal{C}_{\delta}(\mathbf{P}, \overline{\mathbf{P}})=\left\{\left(R_{1}, R_{2}\right): R_{1}\right. & \leq \sum_{i=1}^{T} \frac{1}{2} \log \left(1+P_{i}\right) \\
R_{2} & \leq \sum_{i=1}^{T} \frac{1}{2} \log \left(1+\bar{P}_{i}\right) \\
R_{1}+R_{2} & \left.\leq \sum_{i=1}^{T} \frac{1}{2} \log \left(1+\bar{P}_{i}+P_{i}\right)\right\}
\end{aligned}
$$

For each feasible $(\mathbf{P}, \overline{\mathbf{P}}, \boldsymbol{\delta})$ the region is a pentagon. The capacity region of the Gaussian MAC with energy transfer is the union over all feasible power allocations and energy transfer profiles:

$$
\mathcal{C}(\mathbf{E}, \overline{\mathbf{E}})=\bigcup_{(\delta, \mathbf{P}, \overline{\mathbf{P}}) \in \mathcal{F}} \mathcal{C}_{\delta}(\mathbf{P}, \overline{\mathbf{P}})
$$

where $\mathcal{F}$ is given in (1). This region is shown in Fig. 4.

\section{CAPacity Region of the Gaussian MAC}

In this section, we characterize the capacity region of the Gaussian MAC with one-way energy transfer. First, we note that the capacity region is convex.

Lemma $4 \mathcal{C}(\boldsymbol{E}, \overline{\boldsymbol{E}})$ is a convex region.

Since the region is convex, each boundary point is a solution to $\max _{\mathbf{R} \in \mathcal{C}^{M}} \boldsymbol{\theta} \mathbf{R}$ [18] for some $\boldsymbol{\theta}=\left[\theta_{1}, \theta_{2}\right]$. We examine two cases separately, $\theta_{1} \geq \theta_{2}$ and $\theta_{1}<\theta_{2}$.

\section{A. $\theta_{1} \geq \theta_{2}$}

We show that when $\theta_{1} \geq \theta_{2}$, no energy transfer from user 1 to user 2 is needed. Note that as $\theta_{1} \geq \theta_{2}$, the boundary points between 1, 2 and 3 in Fig. 4 are found by solving the

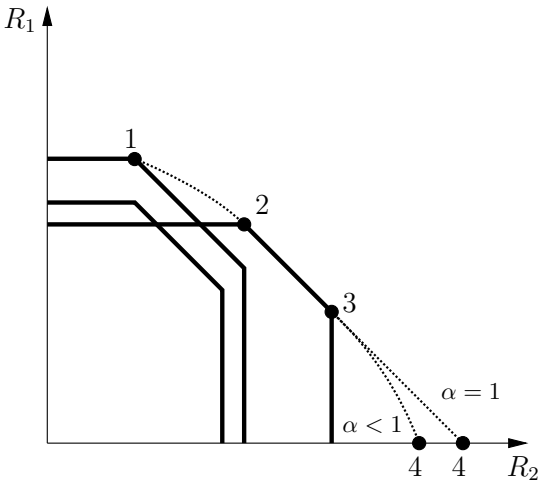

Fig. 4. Capacity region of the Gaussian MAC.

following problem:

$$
\begin{aligned}
\max _{\bar{P}_{i}, P_{i}, \delta_{i}} & \sum_{i=1}^{T}\left(\theta_{1}-\theta_{2}\right) \frac{1}{2} \log \left(1+P_{i}\right)+\theta_{2} \frac{1}{2} \log \left(1+\bar{P}_{i}+P_{i}\right) \\
\text { s.t. } & (\boldsymbol{\delta}, \mathbf{P}, \overline{\mathbf{P}}) \in \mathcal{F}
\end{aligned}
$$

The problem in (23) is a convex optimization problem and the corresponding KKT conditions are:

$$
\begin{array}{rr}
-\frac{\theta_{1}-\theta_{2}}{1+P_{i}}-\frac{\theta_{2}}{1+P_{i}+\bar{P}_{i}}+\sum_{k=i}^{T} \mu_{k}=0, & \forall i \\
-\frac{\theta_{2}}{1+P_{i}+\bar{P}_{i}}+\sum_{k=i}^{T} \eta_{k} & =0, \quad \forall i \\
\sum_{k=i}^{T} \mu_{k}-\alpha \sum_{k=i}^{T} \eta_{k}+\sum_{k=i}^{T} \gamma_{k}-\rho_{i} & =0, \quad \forall i
\end{array}
$$

Since $\theta_{1} \geq \theta_{2}$, from (24)-(25), we have $\sum_{k=i}^{T} \mu_{k} \geq \sum_{k=i}^{T} \eta_{k}$, which is satisfied with equality iff $\theta_{1}=\theta_{2}$. This together with (26) implies that $\rho_{i}-\sum_{k=i}^{T} \gamma_{k} \geq 0$, which is satisfied with equality iff $\theta_{1}=\theta_{2}$ and $\alpha=1$. Therefore, unless we have exactly $\theta_{1}=\theta_{2}$ and $\alpha=1$, then we must have $\rho_{i}>0$ for all $i$. This together with the complementary slackness conditions in (11) implies that we must have $\delta_{i}=0$ for all $i$, i.e., no energy transfer is needed. However, when $\theta_{1}=\theta_{2}$ and additionally if $\alpha=1$, then there may exist multiple different optimal energy transfer profiles, including the one with no energy transfer.

Since energy transfer is not needed, optimal power control policies for the two users are the same as those in the energy harvesting MAC with no energy transfer and can be found by the generalized backward directional water-filling algorithm described in [9]. That is, the capacity region boundary from point 1 to point 3 in Fig. 4 is found by the algorithm in [9]. Specifically, for $\theta_{1}=\theta_{2}$, we have $\eta_{k}=\mu_{k}$ for all $k$ and the sum-rate optimal power policies are obtained by applying single-user directional water-filling algorithm to the sum of the energy profiles of the users [9].

\section{B. $\theta_{1}<\theta_{2}$}

Here, we consider the remaining parts of the boundary, namely the points from point 3 to point 4 in Fig. 4 . In this 
case, we need to solve the following optimization problem:

$$
\begin{aligned}
\max _{\bar{P}_{i}, P_{i}, \delta_{i}} & \sum_{i=1}^{T}\left(\theta_{2}-\theta_{1}\right) \log \left(1+\bar{P}_{i}\right)+\theta_{1} \log \left(1+\bar{P}_{i}+P_{i}\right) \\
\text { s.t. } & (\boldsymbol{\delta}, \overline{\mathbf{P}}, \mathbf{P}) \in \mathcal{F}
\end{aligned}
$$

which is a convex optimization problem and the corresponding KKT conditions are:

$$
\begin{array}{rr}
-\frac{\theta_{1}}{1+P_{i}+\bar{P}_{i}}+\sum_{k=i}^{T} \mu_{k}=0, & \forall i \\
-\frac{\theta_{2}-\theta_{1}}{1+\bar{P}_{i}}-\frac{\theta_{1}}{1+P_{i}+\bar{P}_{i}}+\sum_{k=i}^{T} \eta_{k}=0, & \forall i \\
\sum_{k=i}^{T} \mu_{k}-\alpha \sum_{k=i}^{T} \eta_{k}+\sum_{k=i}^{T} \gamma_{k}-\rho_{i}=0, & \forall i
\end{array}
$$

We do not have an analytical closed form solution for (28)(30). Since (27) is a convex optimization problem, standard numerical methods for convex optimization may be employed. We find that the solution of (27) has a simple form in some special cases, which we investigate next.

When $\alpha=1$, we find that the optimal solution of (27) requires all the energy of user 1 transferred to user 2 . To verify this fact, we note from (28)-(29) that $\eta_{T}>\mu_{T}$, since $\theta_{2}>\theta_{1}$. Combining this with (30), we obtain $\gamma_{T}-\rho_{T}>0$. Note that if $\sum_{i=1}^{T} \delta_{i}<\sum_{i=1}^{T} E_{i}$, then $\gamma_{T}=0$ and hence $\rho_{T}<0$, which is not possible. Thus, in the optimal solution, we must have $\sum_{i=1}^{T} \delta_{i}=\sum_{i=1}^{T} E_{i}$. Therefore, user 1 should not transmit any data, and instead should transfer all of its energy to user 2 by the end of $T$ slots. This policy corresponds to point 4 in Fig. 4. On the other hand, sum-rate optimal point, point 3 , achieves the same throughput as point 4 . This implies that when $\alpha=1$, points 2, 3 and 4 in Fig. 4 lie on the $45^{\circ}$ line. In particular, the optimal throughput of user 2 , which is obtained by singleuser throughput maximization subject to harvested energies of user 2 plus the harvested energies of user 1, coincides with the optimal sum-throughput.

When $\alpha<1$, points 2, 3 and 4 in Fig. 4 are not on the same line. However, we observe that when $\frac{\theta_{2}}{\theta_{1}}$ is sufficiently large, user 1 transfers all of its energy to user 2. In order to verify this claim, we note that, if user 1 transfers some but not all of its energy at the end of $T$ slots, then $\gamma_{T}=0$. In this case, from (28)-(30) and as $\rho_{T} \geq 0$, we have

$$
\frac{1+\bar{P}_{T}}{1+\bar{P}_{T}+P_{T}} \geq \frac{\alpha\left(\theta_{2}-\theta_{1}\right)}{(1-\alpha) \theta_{1}}
$$

Since $\frac{1+\bar{P}_{T}}{1+\bar{P}_{T}+P_{T}}<1$, we conclude that if $\frac{\alpha\left(\theta_{2}-\theta_{1}\right)}{(1-\alpha) \theta_{1}} \geq 1$, then (31) cannot be satisfied which forces all of the energy of user 1 to be transferred to user 2 so that $\gamma_{T}>0$. Note that $\frac{\alpha\left(\theta_{2}-\theta_{1}\right)}{(1-\alpha) \theta_{1}} \geq$ 1 is equivalent to $\frac{\theta_{2}}{\theta_{1}} \geq \frac{1}{\alpha}$. Hence, if $\frac{\theta_{2}}{\theta_{1}} \geq \frac{1}{\alpha}$, in the optimal solution, user 1 transfers all of its energy to user 2 . When $1 \leq \frac{\theta_{2}}{\theta_{1}} \leq \frac{1}{\alpha}$, some non-zero portion of the first user's energy may need to be transferred to the second user in the optimal solution.

\section{CONCLUSIONS}

In this paper, we considered the Gaussian TWC and the Gaussian two-user MAC under energy harvesting and one-way wireless energy transfer conditions. For the Gaussian TWC, we showed that a generalized two-dimensional directional water-filling algorithm, which distributes the overall harvested energy optimally over the time and user dimensions subject to energy causality constraints achieves the boundary of the capacity region. For the Gaussian two-user MAC, with energy transfer from the first user to the second user, we showed that, if the first user has higher priority over the second user, then energy transfer is not needed. In addition, when the second user's priority is sufficiently high, the first user must transfer all of its energy to the second user.

\section{REFERENCES}

[1] B. Gurakan, O. Ozel, J. Yang, and S. Ulukus, "Energy cooperation in energy harvesting wireless communications," in IEEE ISIT, July 2012.

[2] A. Sendonaris, E. Erkip, and B. Aazhang, "User cooperation diversity. Part I. System description,” IEEE Trans. Comm., vol. 51, pp. 1927 1938, November 2003

[3] J. Yang and S. Ulukus, "Optimal packet scheduling in an energy harvesting communication system," IEEE Trans. Comm., vol. 60, pp. 220-230, January 2012.

[4] K. Tutuncuoglu and A. Yener, "Optimum transmission policies for battery limited energy harvesting nodes," IEEE Trans. Wireless Comm., vol. 11, pp. 1180-1189, March 2012.

[5] O. Ozel, K. Tutuncuoglu, J. Yang, S. Ulukus, and A. Yener, "Transmission with energy harvesting nodes in fading wireless channels: Optimal policies," IEEE Jour. on Selected Areas in Commun., vol. 29, pp. 1732 1743, September 2011.

[6] J. Yang, O. Ozel, and S. Ulukus, "Broadcasting with an energy harvesting rechargeable transmitter,' IEEE Trans. Wireless Comm., vol. 11, pp. 571-583, February 2012.

[7] M. A. Antepli, E. Uysal-Biyikoglu, and H. Erkal, "Optimal packet scheduling on an energy harvesting broadcast link," IEEE Jour. on Selected Areas in Commun., vol. 29, pp. 1721-1731, September 2011.

[8] O. Ozel, J. Yang, and S. Ulukus, "Optimal broadcast scheduling for an energy harvesting rechargeable transmitter with a finite capacity battery," IEEE Trans. Wireless Comm., vol. 11, pp. 2193-2203, June 2012.

[9] J. Yang and S. Ulukus, "Optimal packet scheduling in a multiple access channel with energy harvesting transmitters," Journal of Communications and Networks, vol. 14, pp. 140-150, April 2012.

[10] K. Tutuncuoglu and A. Yener, "Sum-rate optimal power policies for energy harvesting transmitters in an interference channel," Journal of Communications and Networks, vol. 14, pp. 151-161, April 2012.

[11] C. Huang, R. Zhang, and S. Cui, "Throughput maximization for the Gaussian relay channel with energy harvesting constraints," IEEE Jour. on Selected Areas in Commun., submitted, September 2011. Available at [arXiv: 1109.0724].

[12] D. Gunduz and B. Devillers, "Two-hop communication with energy harvesting," in IEEE CAMSAP, December 2011.

[13] K. Tutuncuoglu and A. Yener, "Communicating using an energy harvesting transmitter: Optimum policies under energy storage losses," IEEE Trans. Wireless Comm., submitted August 2012, available at [arXiv:1208.6273].

[14] O. Orhan, D. Gunduz, and E. Erkip, "Throughput maximization for an energy harvesting communication system with processing cost," in IEEE ITW, September 2012.

[15] T. S. Han, "A general coding scheme for the two-way channel," IEEE Trans. Inform. Theory, vol. 30, pp. 35-44, January 1984.

[16] S. Boyd and L. Vandenberghe, Convex Optimization. United Kingdom: Cambridge University Press, 2004.

[17] T. Cover and J. Thomas, Elements of Information Theory. Wiley Series in Telecommunications and Signal Processing, John Wiley \& Sons, 2006.

[18] D. Tse and S. Hanly, "Multiaccess fading channels - Part I: Polymatroid structure, optimal resource allocation and throughput capacities," IEEE Trans. Inf. Theory, vol. 44, pp. 2796-2815, November 1998. 